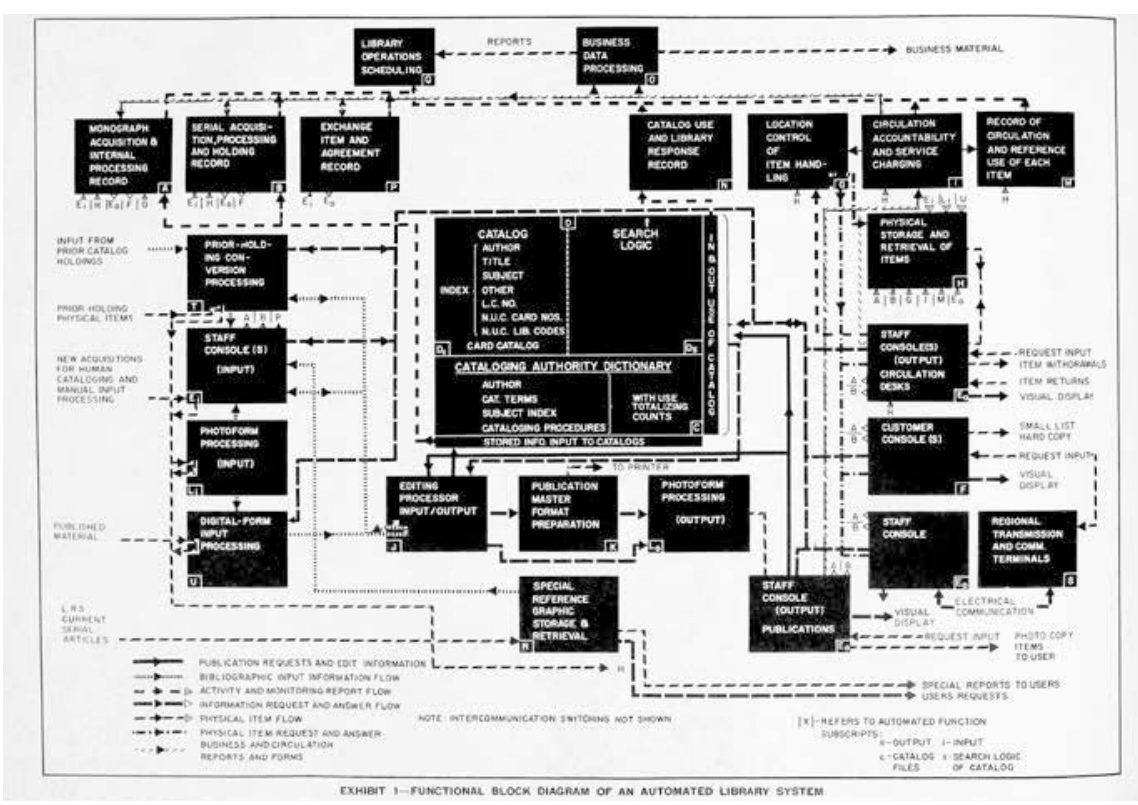

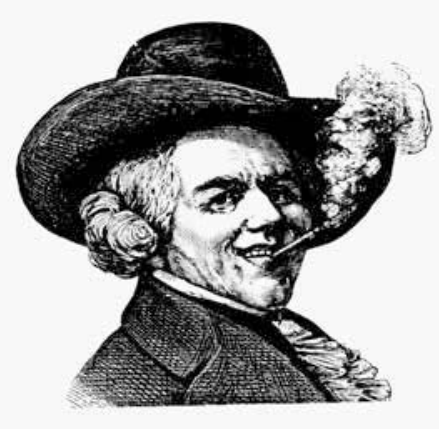

Smoke 'en!

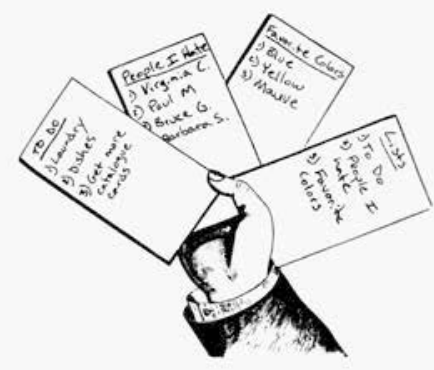

Make 1ists.

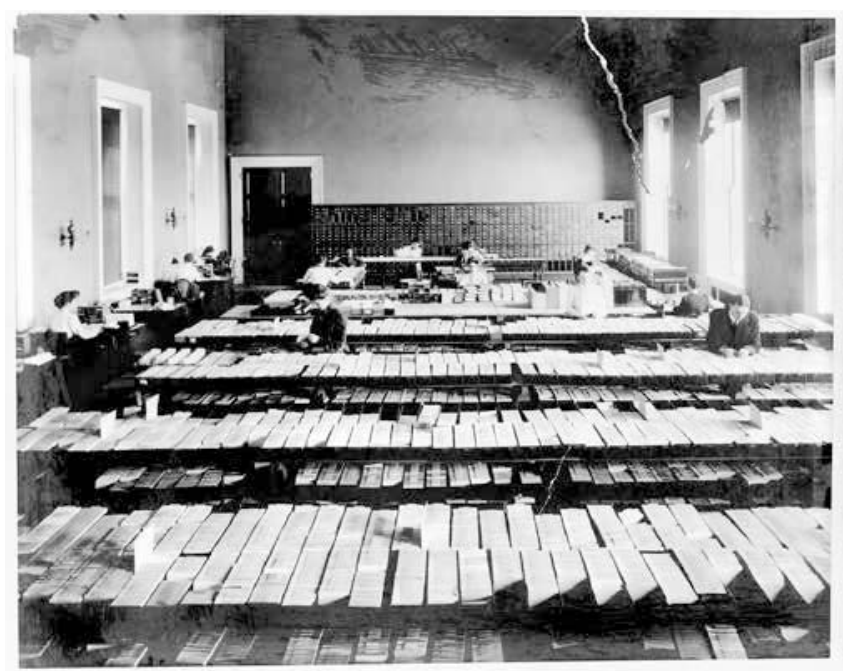

V.I.n.r. Aus: 101 Uses for a Dead Catalogue Card (1985), Blockdiagramm eines automatisierten Bibliothekssystems für die Library of Congress (1963), Zettelkatalog in der Library of Congress zu Beginn des 2o. Jahrhunderts 


\title{
WO BLEIBT DER KATALOG?
}

\author{
von MICHAEL DOMINIK HAGEL
}

1 Where is the catalogue?, in: Website der Universität Utrecht, 1.8.2018, www.uu.nl/en/news/where-isthe-catalogue (26.11.2019).

2 What is WorldCat?, in: Website von OCLC WorldCat, www.worldcat. org/whatis/ (10.1.2019).

3 Vgl. Uwe Jochum: Die Idole der Bibliothekare, Würzburg 1995, 31-53.
«Where is the catalogue?», betitelte die Universität Utrecht einen Blogpost, der im Sommer 2018 online ging. ${ }^{1}$ Die Bibliothek informiert darin über die Abschaltung der Suchfunktion des eigenen Katalogs. Bestände, die bislang über den häuslichen Online Public Access Catalogue (OPAC) auffindbar waren, können - und müssen - nunmehr über WorldCat gesucht werden. Der Verbundkatalog WorldCat ist eine bibliografische Datenbank, die mehrere Milliarden Medieneinheiten verzeichnet und nach gut begründeten Angaben der Betreiberfirma OCLC das größte Netzwerk für Bibliotheksangebote ist. ${ }^{2}$ Wo der Verbundkatalog als Sucheinstieg dient, findet die Bibliotheksrecherche nicht mehr in der lokalen elektronischen Umgebung etwa des universitätseigenen Rechenzentrums statt, in der von Bibliothekar_innen akribisch gepflegte bibliografische Metadaten die Leser_innen zu der von ihnen gesuchten Information führen, sondern nimmt sie den Umweg über die library cloud.

Bibliotheksnutzer_innen mag eine knappe Notiz wie die der Utrechter Bibliothek wenig Sorgen bereiten, sofern ihnen noch nicht gegenwärtig ist, dass der Schritt für sie die zwangsweise Umstellung lange eingeübter und internalisierter Recherchegewohnheiten bedeutet. Bibliothekar_innen dürften solchen Nachrichten mit weniger Gelassenheit begegnen, bedeutet doch das Ende des eigenen Katalogs die Trennung von einem sorgsam und bisweilen liebevoll gepflegten Objekt. ${ }^{3}$ Der bibliothekarische Jargon hat einen eigenen Terminus für den Abschied von jenem Werkzeug, das zwischen Bibliothekssammlung und Bibliothekspublikum vermittelt: Wo von <Katalogabbruch〉 gesprochen wird, ist klar, dass es sich um eine Zäsur handelt, die nicht nur über die einzelne Bibliothek, sondern auch über die Institution Bibliothek hinausreicht.

Katalogabbrüche markieren fundamentale Transformationen in der Konfiguration der Informationsinfrastruktur und sind damit Teil von Mediengeschichten, die Informationsverhalten, technische Apparaturen und Konzeptionen von Information verknüpfen. Der Katalog ist aus dieser Perspektive eine 
neuralgische Schnittstelle innerhalb der Bibliothek, ein Grenzobjekt, in dem sich Vorstellungen des richtigen Umgangs mit Information materialisieren, Potenziale und Schranken technischer Lösungen sichtbar und Rahmenbedingungen für Praktiken der Informationsbewältigung gesetzt werden. Diese Konstellation lässt sich anhand der Geschichte von Bibliothekskatalogen und Katalogisierungspraktiken nachvollziehen. ${ }^{4}$

Eine solche Geschichte kann man - guten Willen vorausgesetzt - mit Keilschrifttafeln beginnen lassen. ${ }^{5} \mathrm{Zu}$ ihr gehören klösterliche Bandkataloge oder die Spielkarten, auf deren Rückseite im revolutionären Frankreich die konfiszierten Buchbestände erfasst wurden und in denen man die Ursprünge des Zettelkatalogs ausgemacht hat. Der mit der Sattelzeit einsetzenden (und längst verstrichenen) Epoche des Zettelkastens eignet gar ein nostalgisches Angebot: Reprografien der schönsten Katalogzettel der Library of Congress sind samt Pappmodell eines Zettelkastens im Handel zu erwerben; die Bibliothekstechnik des normierten Karteisystems wurde - in Deutschland - gar zur «Maschine der Phantasie» erklärt. ${ }^{6}$ Aber auch die aufschlussreichsten und ambitioniertesten Arbeiten einer solchen Kataloggeschichte adressieren nur mittelbar die epistemischen Verschiebungen, in die sich die Migration der Bibliotheksdaten in die Cloud einreiht. ${ }^{7}$ Selbst eine Geschichte früherer elektronischer Kataloge, die seit den I970er Jahren Einzug in Bibliotheken nahmen, ist nur rudimentär vorhanden. ${ }^{8}$

Freilich ist die Gegenwart der Kataloge geprägt von einer ubiquitären Banalität des Suchens und Findens, jenes operativen Schemas also, das als «Inbegriff der Bibliothek» beschrieben wurde. ${ }^{9}$ Entsprechend ist die Bibliotheksrecherche längst abhängig geworden von algorithmengesteuerten Suchmaschinentechnologien..$^{10}$ Anstatt wie einst in der vergleichsweise übersichtlichen Masse von nach Regelwerken erzeugten Metadaten des Bibliotheksbestands zu suchen, recherchiert der_die Bibliotheksbenutzer_in nunmehr mittels für ihn_sie ebenso wie für das Bibliothekspersonal undurchsichtigen Mechanismen in Megaindices, die von kommerziellen Anbietern bereitgestellt werden und bibliothekarische Erschließungstraditionen in Frage stellen. ${ }^{11}$ Diese Veränderungen in der Suche sind auch Antworten auf die stetig wachsende Masse wissenschaftlicher Information in einer globalen Forschungslandschaft: WorldCat verzeichnet 464 Millionen bibliografische Einträge, die der schlecht vorstellbaren Zahl von 2,853 Milliarden verfügbaren Medieneinheiten entsprechen, ${ }^{12}$ wogegen die r67 Millionen Medieneinheiten (davon kaum 40 Millionen Bücher) der Library of Congress oder die ${ }_{1} 8_{5}$ Millionen, die deutsche Hochschulen gemeinsam bereitstellen, zwergenhaft wirken. ${ }^{13}$ Die Größenordnungen des von der Firma OCLC (I967 als Ohio College Library Center gegründet) betriebenen WorldCat oder des von der Firma ExLibris (hervorgegangen aus einem internen Projekt der Hebräischen Universität Jerusalem) angebotenen Central Discovery Index, der über 3 Milliarden Einträge bereithält, sprengen die Kapazitäten nicht nur von physischen Zettelkästen, sondern auch der dahinter stehenden Logik.
4 Vgl. Eugene R. Hanson, Jay E. Daily: Catalogs and Cataloging: History [ELIS Classic], in: John D. McDonald, Michael Levine-Clark (Hg.): Encyclopedia of Library and Information Sciences, London, New York 2018, 743-779.

5 The Library of Congress (Hg.): The Card Catalog: Books, Cards, and Literary Treasures, San Francisco 2017.

6 Heike Gfrereis, Ellen Strittmatter (Hg.): Zettelkästen. Maschinen der Phantasie, Marbach am Neckar 2013.

7 Z. B. Ronald Day: Indexing It All. The Subject in the Age of Documentation, Information, and Data, Cambridge 2014.

8 Vgl. Markus Krajewski: Die Bibliothek als Meta-Medium, in: Konrad Umlauf, Stefan Gradmann (Hg.): Handbuch Bibliothek. Geschichte, Aufgaben, Perspektiven, Stuttgart, Weimar 2012, 81-89; ders.: Der Diener. Mediengeschichte einer Figur zwischen König und Klient, Frankfurt/M. 2010, 201-208.

9 Nikolaus Wegmann: Im Labyrinth. Über die (Un-)Möglichkeit der Bibliothek als Qualitätsmedium, in: Bibliothek - Forschung und Praxis, Jg. 42, Nr. 2, 2018, 370-378, hier 376, doi.org/10.1515/bfp-2018-0047. Vgl. auch ders.: Bücherlabyrinthe. Suchen und Finden im alexandrinischen Zeitalter, Köln 2000.

10 Jutta Haider, Olof Sundin: Invisible Search and Online Search Engines. The Ubiquity of Search in Everyday Life, London, New York 2019. Ken Hillis, Michael Petit, Kylie Jarrett: Google and the Culture of Search, New York 2013.

11 David Weinberger: Everything is Miscellaneous. The Power of the New Digital Disorder, New York 2007.

12 Inside WorldCat, in: Website von OCLC WorldCat, www.oclc. org/en/worldcat/inside-worldcat.html (2.12.2019).

13 Jürgen Seefeldt: Hochschulund Universitätsbibliotheken, in: Bibliotheksportal.de, 1.8.2017, bibliotheksportal.de/informationen/ bibliothekslandschaft/hochschul-unduniversitaetsbibliotheken/ (4.12.2019). 
14 Vgl. Markus Krajewski: Zettelwirtschaft. Die Geburt der Kartei aus dem Geiste der Bibliothek, Berlin 2002.

$15 \mathrm{Vgl}$. Rafael Ball: Datenräume. Bibliotheksverbünde der Zukunft - welche Bibliotheksverbünde?, in: Zeitschrift für Bibliothekswesen und Bibliographie, Bd. 61, 2014, 267-270.

16 Wegmann: Im Labyrinth, 377.
Denn mit der algorithmenbasierten Suche in miteinander vernetzten, heterogenen Informationsbeständen ist die Epoche des Zettelkastens, wie sie in den lokalen elektronischen Katalogen noch herrschte, tatsächlich an ihr Ende gekommen. Die mit dem Aufgehen lokaler Kataloge in Verbundsystemen einhergehenden Transformationen betreffen nicht nur die Größenordnung, sondern markieren eine fundamentale Umstellung der Grundlagen der Suche. Bibliografische Dateiformate wie das Maschinelle Austauschformat für Bibliotheken - so der volle Name des in Deutschland 1973 veröffentlichten Standards MAB - wurden sorgsam nach dem intellektuellen Format der Karteikästen modelliert. Entwicklungen solcher Normen wurden seitens der Library of Congress ab den I950er Jahren vorbereitet, seit den I96oer Jahren ist MachineReadable Cataloging (MARC) im Einsatz. Computer hielten Einzug in die immer gigantischer gewordenen (Zettel-)Katalogsäle. Nach und nach wurden elektronische Datenbanken, die die Leistung der Zettelkataloge durch brachiale Performanz übertrafen, aufgebaut und ineinander integriert. Die elektronische Formatierung nach dem Muster des Katalogzettels war das Fundament der lokalen rechnergestützten Kataloge und zugleich deren Limitierung im Hinblick auf die Vernetzung mit nicht mit bibliothekarischem Eifer gepflegten Daten. Unter den Vorzeichen eines neuen, die säuberliche tabellarische Ordnung der elektronischen Datenverarbeitung sprengenden Computerzeitalters entsteht eine genuin neue Spielart des Katalogs - mit weitreichenden Implikationen für die wissenschaftliche Informationsinfrastruktur.

Wenn die bibliothekarische Informationsverwaltung in der Epoche des Zettelkastens wegweisend für die Datenverarbeitung insgesamt war, ${ }^{14}$ so zeigt sich mit Blick auf die aktuelle Lage des Katalogs eine widersprüchliche Konstellation. Kommerzielle Anbieter setzen vor allem außerhalb des bibliothekarischen Bereichs Standards des Suchens und Findens, die mit den - für Außenstehende oft schwer nachzuvollziehenden - bibliothekarischen Ansprüchen an die Qualität von (Meta-)Daten kaum kompatibel sind. ${ }^{\mathbf{1 5}}$ Suchmaschinentechnologien, die mittels Ranking-Algorithmen Orientierung in Datenmassen schaffen sollen, wurden nicht auf Zwecke der wissenschaftlichen Informationsversorgung mit ihren vom Alltagsgebrauch abweichenden Ansprüchen an precision und recall hin entwickelt. Für die Bibliothek stellt sich damit die Frage nach ihren Standards nicht nur auf der technischen Ebene des Datenformats, sondern vor allem in Bezug auf die Qualität bibliothekarischer user experience.

Fantasien des punktgenauen und trennscharfen information retrieval in bibliothekarisch fruchtbar gemachten Big Data steht die Praxis durchwachsener Such- und Findeerfahrungen mit zeitgenössischen Bibliothekskatalogen gegenüber. Auf absehbare Zeit ist damit die auf einer gewissen Hermetik aufbauende Kreativitätsprämie der Bibliotheken - von Nikolaus Wegmann als «eigentliche Stärke der Bibliothek» ausgemacht ${ }^{16}$ - gesichert. Wie sich um den Komplex 〈Katalog〉 gegenwärtig harte technische Fragen nach 
Datenformaten und Kodierungen mit dem täglichen Geschäft der Wissenschaftler_innen verzahnen, zeigt exemplarisch die Auskunft eines Neogräzisten auf die Frage nach seinen Erfahrungen mit dem megaindexbasierten Bibliothekskatalog: In seinem Fach verstünde man den aktuellen Katalog als Einladung, Literatur am Regal zu suchen. Die Anekdote trifft insofern ins Zentrum des Problems, als sich im Zusammenhang mit nicht-lateinischen Schriften besonders deutlich zeigen lässt, wie fragil, verzerrt und fehleranfällig das Konzept einer vorgeblich globalen library cloud ist. ${ }^{17}$ Die Antwort auf die Frage danach, wo der Katalog ist, weist jedenfalls in die Datenwolke. Deren unstrittige Potenziale - etwa im Sinne einer Verzahnung von Bibliothekskatalogen mit dem semantischen Web - müssen die Balance mit der grundlegenden Nachweisfunktion des Katalogs noch finden, gerade dort, wo die Suche am Regal kein Thema mehr ist.
17 Vgl. Domenico Fiormonte: Towards a Cultural Critique of the Digital Humanities, in: Historical Social Research, Bd. 37, Nr. 3, 2012, $59-76$. 